\title{
Insulin resistance and other risk factors of cardiovascular disease in abnormal uterine bleeding
}

\section{Hannah Shehata}

Loma Linda University Health: Loma Linda University Adventist Health Sciences Center

\section{Abigail Berry}

Loma Linda University School of Medicine

\section{Christopher Riba}

Loma Linda University School of Medicine

Andrea Cristine Salcedo ( $\nabla$ drandreasalcedo@gmail.com )

Loma Linda University Health: Loma Linda University Adventist Health Sciences Center https://orcid.org/0000-0002-4803-3587

\section{Research}

Keywords: abnormal uterine bleeding, insulin resistance, cardiovascular disease risk, obesity, waist hip ratio, fasting insulin

Posted Date: July 1st, 2021

DOI: https://doi.org/10.21203/rs.3.rs-604656/v1

License: (c) (1) This work is licensed under a Creative Commons Attribution 4.0 International License. Read Full License 


\section{Abstract \\ Background}

Studies indicate the presence of cardiovascular disease risk factors among patients with uterine fibroids and polycystic ovarian syndrome. Investigators were interested in examining the prevalence of cardiovascular disease risk factors and the independent predictors of insulin resistance (IR) among women with abnormal uterine bleeding (AUB).

\section{Methods}

This observational study examined 77 premenopausal subjects with AUB. Their medical history, body mass index, waist hip ratio, fasting insulin level, hemoglobin A1C, creatinine level, and lipid panel were evaluated. The participants were then subdivided using insulin levels and further compared. IR was defined at two different fasting insulin levels: $>10 \mu \mathrm{U} / \mathrm{mL}$ and $>15 \mu \mathrm{U} / \mathrm{mL}$.

\section{Results}

Of the 77 data sets analyzed, $91 \%$ of participants were current or former smokers, $62 \%$ had a waist hip ratio $>0.80,45 \%$ were obese, and the majority of participants had dyslipidemia, with high total cholesterol $(68 \%)$ and elevated LDL (38\%) being the most prevalent. $66 \%$ of participants were insulin resistant as defined by the lower fasting insulin and $38 \%$ were insulin resistant as defined by the upper fasting insulin level. At the lower cutoff, the insulin resistant group was significantly more likely to have a waist hip ratio $>0.8$, be obese, and have low HDL levels. At the upper fasting insulin cutoff, the insulin resistant group was significantly more likely to have low HDL levels, elevated triglyceride levels, a waist hip ratio $>0.8$, and be obese. Univariate analysis showed statistically significant associations between IR in women with AUB and body mass index, waist hip ratio, and HDL for both fasting insulin cutoffs. Multivariate analysis using the lower fasting insulin cutoff revealed that body mass index and waist hip ratio were significant independent predictors of IR in a woman with AUB.

\section{Conclusions}

This study reveals the prevalence of IR and other predictors of cardiovascular disease are greater in women with AUB in this population than the general population. Body mass index and waist hip ratio are independent predictors of IR in women with AUB. Low HDL levels, elevated triglyceride levels, and ethnicity are also significantly associated with IR in women with AUB depending on the definition used.

\section{Background}


Abnormal uterine bleeding (AUB) is one of the most common reasons reproductive-aged women seek a gynecologic consult. It affects up to 35 percent of women in their lifetime and comprises up to 70 percent of outpatient gynecology visits each year. ${ }^{1-2}$ AUB is a broad term that is classified by its causes using the PALM-COEIN system and it is often paired with descriptive terms denoting heavy versus intermenstrual bleeding. ${ }^{1}$

Insulin is a key anabolic hormone involved in multiple metabolic pathways in the body, including glucose uptake and lipid storage, cell growth and proliferation in the body, and nitric oxide (NO) synthesis by endothelial cells. ${ }^{3-5}$ Insulin resistance (IR) occurs when increasingly larger amounts of insulin are required to regulate the body's metabolic pathways, and, even in the face of these increasing insulin levels, cellular activity is diminished compared to a normal person. ${ }^{3-5} \mathrm{IR}$ is a multifactorial disease that has become an epidemic in the United States (US). Although the number of adults affected by IR is not known, one in three adults in the US have prediabetes and an additional 10.5 percent of adults have diabetes mellitus type 2 (DM2). ${ }^{3,6}$ These numbers are expected to double in the next 10 years since almost 90 percent of Americans having risk factors for IR. ${ }^{6}$

Although angiogenesis is a relatively infrequent event in most adult tissues, it occurs repeatedly in the uterus in order to support the monthly cycle of growth, shedding, and repair of the endometrial lining. ${ }^{7}$ Both the process of angiogenesis and endometrial repair are tightly regulated by estrogen and progesterone, which involve NO, platelets, immune cells, and multiple growth factors. ${ }^{2,7-10}$ Decreased NO production, due to IR, results in an increase in the shear stress on endothelial cells due to diffuse vasoconstriction. ${ }^{3,5}$ Moreover, decreased NO, hyperglycemia, and the state of IR itself promote the release of inflammatory cytokines and free radical formation, which leads to further endothelial damage, surrounding tissue hypoxia, and, eventually, cellular apoptosis. ${ }^{3-5}$ This increases vascular permeability, which is worsened by high levels of circulating glucose also directly damaging the glycocalyx and endothelial cells through non-enzymatic glycation. ${ }^{3,5,11}$ Together, these vascular changes allow substrates to leave the blood and form plaques in the tunica intima layer of the blood vessel. ${ }^{5}$ This thickening of the blood vessels leads to increased vascular resistance, chronic low-grade inflammation, and atherosclerosis. ${ }^{3,5}$

Applying this knowledge to the uterus, chronic low-grade inflammation likely reduces the spiral arteries' ability to meet the high metabolic demands of the endometrium. This leads to an overexpression of angiogenic cytokines and dysfunctional angiogenesis with excessive uterine vessel formation and dilation in an attempt to increase the amount of uterine blood flow. ${ }^{7-8,10,12}$ It also results in functionally impaired proliferative and secretory phases of the menstrual cycle where the endometrial lining is not properly repaired during cyclical shedding. Instead, the same inflammatory cytokines and fibrosispromoting growth factors seen in atherosclerotic disease encourage smooth muscle metaplasia and scarring in the uterus. ${ }^{12-19}$ 
Atherosclerosis is a widely accepted cause of numerous microvascular and macrovascular diseases that are significantly associated with IR. ${ }^{3-5,20-23}$ Multiple studies have demonstrated that women with fibroids and polycystic ovarian syndrome (PCOS) are more likely to have risk factors for cardiovascular disease, but AUB is not currently thought of as an atherosclerotic-related end organ disease. Furthermore, few studies have examined these risk factors in women with AUB as a whole (referred to in this paper as overall AUB). ${ }^{16-18,24-31}$ The aim of the present study is to describe the prevalence of insulin resistance and other predictors of cardiovascular disease among women with overall AUB.

\section{Methods}

\section{Study Population}

This was a cross-sectional observational study conducted at Loma Linda University Medical Center's outpatient obstetrics and gynecology clinics. Potential study candidates were all premenopausal females between the ages of 18 and 54 years old who presented with a complaint of AUB between September 2018 and October 2019. A candidate was confirmed eligible for enrollment once the diagnosis of AUB was made in the outpatient clinic setting. Women with a history of gynecologic cancer, prior hysterectomy, prior endometrial ablation, prior tamoxifen use, diabetes mellitus type 1, chronic steroid use, or pregnancy were excluded. There were 117 subjects enrolled with a total of 77 data sets analyzed for this study.

\section{Ethics Statement}

The participants gave voluntary, informed, written consent prior to participating in the study. This research study was approved by the Loma Linda University Institutional Review Board and it complied with all relevant national regulations, institutional policies, and the principles of the Declaration of Helsinki as they relate to human participants. All participant identifiers were replaced with identification codes and the data was handled only by the researchers participating in this study.

\section{Measured Variables}

At the time of enrollment, a thorough history was performed. Each participant had her height, weight, and waist to hip ratio (WHR) measured. Overnight fasting blood samples were then obtained to assess each participant's fasting insulin level (FI), hemoglobin A1C (A1C), creatinine ( $\mathrm{Cr}$ ), and lipid profile. A BMI $\geq$ $30 \mathrm{~kg} / \mathrm{m}^{2}$ was used as the cutoff for obesity and a WHR $>0.80$ was considered abnormal. ${ }^{32-33} \mathrm{IR}$ was assessed using two different FI cutoffs: $>10 \mu \mathrm{U} / \mathrm{mL}$ and $>15 \mu \mathrm{U} / \mathrm{mL}$, which represented both the lower and higher end of the FI cutoffs of prior research. ${ }^{4,22,34-35}$ Abnormal A1C values were defined by the prediabetic cut off of $\geq 5.7 \%$, while abnormal lipid levels were defined by the American Heart Association values of $\mathrm{HDL}<50 \mathrm{mg} / \mathrm{dL}$, total cholesterol levels $>150 \mathrm{mg} / \mathrm{dL}, \mathrm{LDL}>100 \mathrm{mg} / \mathrm{dL}$, and triglycerides levels > $150 \mathrm{mg} / \mathrm{dL} .6,36$

\section{Statistical Analysis}


Statistical analysis was performed using the online statistical software R version 3.5.1. ${ }^{37}$ Assuming 33 percent of the general population is $I R$, the minimum sample size for the present study to have adequate power was calculated to be 36 subjects in each group. ${ }^{6}$ Independent t-test, Mann Whitney $\mathrm{U}$ test, and chisquare test were calculated with the statistical significance set as $P>0.05$. Univariate and multivariate regression analysis were performed using linear regression. Observed associations were expressed as odds ratios with 95 percent confidence intervals.

\section{Results}

Of the 117 participants enrolled, 77 participants completed the measured variables and were included in the analysis. As shown in Table 1, most participants were Hispanic or White females, averaging 37 years of age, with an average of two prior births. Most participants did not have relevant medical or surgical history, except for over $90 \%$ being either current or former smokers. Approximately two-thirds of participants had a WHR $>0.80$ with the average WHR being 0.86 . Almost half of participants were obese with the average BMI being $31.82 \mathrm{~kg} / \mathrm{m}^{2}$. Two-thirds of participants had a $\mathrm{FI}>10 \mu \mathrm{iU} / \mathrm{mL}$ with $38 \%$ having a $\mathrm{FI}>15 \mu \mathrm{iU} / \mathrm{mL}$, but only $22 \%$ of participants had an $\mathrm{A} 1 \mathrm{C} \geq 5.7 \%$. The majority of participants also had dyslipidemia with $68 \%$ having high total cholesterol levels and an additional $38 \%$ of participants having high LDL levels.

Table 2 shows the difference in characteristics between the two groups when IR was defined as $\mathrm{FI}>$ $10 \mu \mathrm{iU} / \mathrm{mL}$. Ethnicity was significantly related to IR with all African Americans and Middle Easterners being classified as IR, while none of the Asian participants were classified as IR $(p=0.027)$. The IR group was significantly more likely to be obese $(60.8 \%$ versus $15.4 \% ; p<0.001)$ and have low HDL levels $(41.2 \%$ versus $19.2 \% ; p=0.03$ ) than the non-IR group. There were no statistical differences between the two groups with regards to the other variables analyzed, but it is worth noting that no participants in the nonIR group had a history of DM2 or elevated triglycerides levels, and more than $62 \%$ of participants in both groups had high total cholesterol levels.

Table 3 depicts the difference in characteristics between the two groups when IR was defined as $\mathrm{FI}>$ $15 \mu \mathrm{iU} / \mathrm{mL}$. The IR group was significantly more likely to be obese $(75.9 \%$ versus $27.1 \% ; p<0.001)$, have low HDL levels ( $48.3 \%$ versus $25 \%$; $p=0.016)$, and have high triglycerides levels $(13.8 \%$ versus $2.1 \% ; p=$ 0.032) compared to the non-IR group. Although there were no statistical differences between the two groups regarding the other variables analyzed, again, no Asian participants were classified as IR, and more than $62 \%$ of participants in both groups had elevated total cholesterol levels.

By univariate regression analysis, factors significantly associated with IR in women with AUB were BMI, WHR, and HDL levels. These associations persisted regardless of the FI level used to define IR (see Table 4). Ethnicity, Cr, triglycerides levels, total cholesterol levels, LDL, history of DM2, history of hypertension, history of migraines, and smoking were not significantly associated with IR in women with AUB in either of the univariate regression analyses. When multivariate regression analysis was performed using $\mathrm{FI}>10 \mu \mathrm{iU} / \mathrm{mL}$ to define IR, BMI (standardized coefficient $\beta: 1.07, \mathrm{P}=0.015$ ) and WHR (standardized 
coefficient $\beta$ : $1.94, P=0.045$ ) were independent predictors of IR in women with AUB. Regression analysis was not performed using $\mathrm{FI}>15 \mu \mathrm{iU} / \mathrm{mL}$.

\section{Discussion}

This study shows the prevalence of IR and other predictors of cardiovascular disease are higher among women from this diverse suburban community with overall AUB (66\%) than among the general population. BMI and WHR were shown to be independent predictors of IR in women with AUB. The odds of a woman with AUB having IR was 7 times higher for every 1 unit increase in BMI over $30 \mathrm{~kg} / \mathrm{m}^{2}$ when confounding for other variables. By the same convention, the odds of a woman with AUB having IR was nearly 4 times higher for every 0.1 unit increase in WHR after 0.8. Low HDL levels, elevated triglycerides levels, and ethnicity were also significantly associated with IR in women with AUB, depending on how IR was defined.

Similar to prior studies on AUB, the present study's participants had a high prevalence of risk factors for cardiovascular disease, including smoking (90\%), obesity (45\%), an elevated WHR (62\%), and dyslipidemia. ${ }^{16,18,24-25,28-31}$ The majority of participants did not have an elevated A1C or a history of hypertension or DM2, but this lack of association has been noted in prior studies on overall AUB as well. ${ }^{28-29}$ Prior studies have found a relationship between AUB and elevated triglycerides levels, but this relationship was only significant in the present study when participants were IR using the higher FI cutoff

of $>15 \mu \mathrm{iU} / \mathrm{mL}^{28-29}$ Interestingly, the significant inverse relationship between IR and HDL levels in women with AUB in the present study was not seen in prior studies on overall AUB. ${ }^{28-29}$ Participants with normal HDL levels, which are considered cardioprotective, were almost 70 percent less likely to be IR. ${ }^{36}$ Additionally, although a significant relationship between BMI and/or WHR and AUB has been reported in multiple prior studies, the relationship between BMI and/or WHR, IR, and AUB has previously only been shown to be significant in prior studies on AUB due to PCOS. ${ }^{16,18,24-25,28-31}$

Some of the strengths of this study were the diverse patient population studied and the broad definition of AUB intentionally used to evaluate the overall relationship between AUB and risk factors for cardiovascular disease. However, this also made it difficult to compare the present study's results to the results of prior studies, which often only evaluated one specific cause of AUB and included less diverse study populations. Using a diverse study population limits how generalizable the present study's results are to more homogenous regions. Furthermore, as the pathophysiology causing AUB in women with irregular cycles compared to those with AUB and regular cycles may be different, the prevalence of the different risk factors for cardiovascular disease may not be the same in these different subsets of women. Additionally, as this was a cross-sectional study, it can only identify associations between IR and other cardiovascular disease risk factors among women with AUB.

Another weakness of this study was the high attrition rate present with $41(35 \%)$ of the participants being excluded from the analysis due to not completing any laboratory data despite regular reminders by the study's investigators. Although still included in the analysis, an additional four (3\%) of the participants 
did not complete the required lipid panel and an additional $16(13.6 \%)$ of the participants did not undergo evaluation of their WHR. This selection bias likely affects how generalizable the results from the present study are to women with AUB even. Finally, attempts to limit investigator bias were made by using retrospective self-reports to diagnose AUB, but prior studies have shown these may not be accurate. ${ }^{38}$

\section{Implications for Research}

Further research is needed on the relationship between ethnicity and IR as ethnicity has been shown to be an important predictor of cardiovascular disease in the general population. ${ }^{39}$ Although ethnicity was shown to be significantly related to IR in women with AUB when IR was defined as FI > $10 \mu \mathrm{iU} / \mathrm{mL}$, it may not have been significantly related to IR using the higher FI cutoff due to a small sample size. A follow-up study is currently underway to compare the prevalence of IR and other cardiovascular disease risk factors in women with AUB to normally menstruating women within this same diverse suburban community.

\section{Conclusions and Implications}

The high prevalence of risk factors for cardiovascular disease seen in these women suggests that AUB may be a sentinel finding for DM2 and cardiovascular disease in women later in life. It is vital for clinicians to screen women from diverse suburban communities with AUB for metabolic syndrome and these other cardiovascular disease risk factors. These patients also need to be educated on lifestyle changes they can make to reduce their risk for cardiovascular disease.

\section{Abbreviations}

AUB

Abnormal uterine bleeding

PALM-COEIN

Polyp, Adenomyosis, Leiomyoma, Metaplasia- Coagulopathy, Ovulatory, Endometrial, latrogenic, Not otherwise specified

IR

Insulin resistance

NO

Nitric oxide

DM2

Diabetes mellitus type 2

PCOS

polycystic ovarian syndrome

$\mathrm{FI}$

Fasting insulin

BMI

Body mass index 
WHR

Waist hip ratio

\section{Declarations}

\section{Ethics approval to participate:}

The participants gave voluntary, informed, written consent prior to participating in the study. This research study was approved by the Loma Linda University Institutional Review Board and it complied with all relevant national regulations, institutional policies, and the principles of the Declaration of Helsinki as they relate to human participants. All participant identifiers were replaced with identification codes and the data was handled only by the researchers participating in this study.

\section{Consent for publication}

Not applicable

\section{Availability of data and materials}

The datasets used and/or analyzed during the current study are available from the corresponding author on reasonable request.

Competing interests

The authors declare they have no competing interests.

\section{Funding}

This study was supported by Loma Linda University Health, Department of Gynecology and Obstetrics.

\section{Authors' contributions}

AS made substantial contributions to the conception, design of the work, acquisition and interpretation of data, and substantively revised the work. HS made substantial contributions to the acquisition and interpretation of data, drafted and substantively revised the work. AB and CR made substantial contributions to the acquisition of data. All authors have approved the submitted version and agree to be personally accountable for the author's own contributions to ensure that questions related to the accuracy or integrity of any part of the work, even ones in which the author was not personally involved, are appropriately investigated, resolved, and the resolution documented in the literature.

\section{Acknowledgements}

The authors would like to thank Jiahao Peng, MD, PhD, and Wendy Shih, DrPH of the Research Consulting Group of Loma Linda University Health, for their contributions to the analysis of the data. 


\section{References}

1. American College of Obstetricians and Gynecologists. Diagnosis of abnormal uterine bleeding in reproductive-aged women. ACOG Practice Bulletin No. 128. Washington DC; 2012.

2. Davis E, Sparzak PB. Abnormal uterine bleeding (dysfunctional uterine bleeding). StatPearls. Treasure Island FL: StatPearls Publishing; 2020. https://www.ncbi.nlm.nih.gov/books/NBK532913/. Accessed February 26, 2020.

3. Ormazabal V, Nair S, Elfeky O, et al. Association between insulin resistance and the development of cardiovascular disease. Cardiovasc Diabetol. 2018;17:122. doi:10.1186/s12933-018-0762-4.

4. Mansyur M, Bakri S, Patellongi I, Rahman I. The association between metabolic syndrome components, low-grade systemic inflammation, and insulin resistance in non-diabetic Indonesian adolescent male. Clin Nutr, 2020; 35. doi:10.1016/j.clnesp.2019.12.001.

5. Cummins I. Primary health challenges \& their root causes. Lecture presented: Scientific \& evolutionary evidence through the Nutrition Network. https://courses.nutrition-network.org. Assessed January 24, 2020.

6. US Department of Health and Human Services. National diabetes statistics report. Atlanta: Centers for Disease Control and Prevention; 2020.

7. Reynolds LP, Grazul-Bilska AT, Redmer DA. Angiogenesis in the female reproductive organs: Pathological implications. Int J Exp Pathol. 2002;83(4):151-63. doi:10.1046/j.13652613.2002.00277.x.

8. Chodankar R, Critchley H. Biomarkers in abnormal uterine bleeding. Biol Reprod. 2019;101(6):115566. doi:10.1093/biolre/ioy231.

9. Ciarmela P, Islam MS, Reis FM, et al. Growth factors and myometrium: Biological effects in uterine fibroid and possible clinical implications. Hum Reprod. 2011;17(6):772-90. doi:10.1093/humupd/dmr031.

10. Berbic M, Fraser IS. Immunology of normal and abnormal menstruation. J Womens Health (Lond). 2013;9(4):387-95. doi:10.2217/whe.13.32.

11. Van den Berg B, Spaan J, Vink H. Impaired glycocalyx barrier properties contribute to enhanced intimal low-density lipoprotein accumulation at the carotid artery bifurcation in mice. J Physiol (Europe). 2009;457:1199-206. doi:10.1007/s00424-008-0590-6.

12. Islam MS, Akhtar MM, Ciavattini A, et al. Use of dietary phytochemicals to target inflammation, fibrosis, proliferation, and angiogenesis in uterine tissues: Promising options for prevention and treatment of uterine fibroids? Mol Nutr Food Res. 2014;58(8):1667-84. doi:10.1002/mnfr.201400134.

13. Protic 0 , Toti $P$, Islam MS, et al. Possible involvement of inflammatory/reparative processes in the development of uterine fibroids. Cell Tissue Res. 2016;364:415-27. Doi:10.1007/s00441-015-2324-3.

14. Wynn TA. Cellular and molecular mechanisms of fibrosis. J Pathol. 2008;214(2):199-210. doi:10.1002/path.2277. 
15. Pardali E, Sanchez-Duffhues G, Gomez-Puerto MC, Ten Dijke P. TGF- $\beta$-induced endothelialmesenchymal transition in fibrotic diseases. Int J Mol Sci. 2017;18(10):2157. doi:10.3390/ijms18102157.

16. Uimari O, Auvinen J, Jokelainen J, et al. Uterine fibroids and cardiovascular risk. Hum Reprod. 2016;31(12):2689-703. doi:10.1093/humrep/dew249.

17. Silver MA, Raghuvir R, Fedirko B, Elser D. Systemic hypertension among women with uterine leiomyomata: Potential final common pathways of target end-organ remodeling. J Clin hypertension. 2005;7(11):664-8.

18. Scicchitano P, Dentamaro I, Carbonara R, et al. Cardiovascular risk in women with PCOS. Int J Endocrinol Metab. 2012;10(4):611-8. doi:10.5812/ijem.4020.

19. Stewart EA, Nowak RA. New concepts in the treatment of uterine leiomyomas. Obstet Gynecol. 1998;92(4):624-7. doi:10.1016/s0029-7844(98)00243-9.

20. Do HD, Lohsoonthorn V, Jiamjarasrangsi W, et al. Prevalence of insulin resistance and its relationship with cardiovascular disease risk factors among Thai adults over 35 years old. Diabetes Res Clin Pract. 2010;89(3):303-8. doi:10.1016/j.diabres.2010.04.013.

21. Baker LD, Cross DJ, Minoshima S, et al. Insulin resistance and Alzheimer-like reductions in regional cerebral glucose metabolism for cognitively normal adults with prediabetes or early type 2 diabetes. Arch Neurololgy. 2011;68(1):51-7. doi:10.1001/archneurol.2010.225.

22. De Boer IH, Katz R, Chonchol MB, et al. Insulin resistance, cystatin $\mathrm{C}$, and mortality among older adults. Diabetes Care. 2012;35(6):1355-60. doi:10.2337/dc11-1657.

23. Wang W, Lo A. Diabetic retinopathy: Pathophysiology and treatments. Int J Mol Sci. 2018;19(6):1816. doi:10.3390/ijms19061816.

24. Laughlin-Tommaso SK, Fuchs EL, Wellons MF, et al. Uterine fibroids and the risk of cardiovascular disease in the Coronary Artery Risk Development in Young Adult Women's study. J Womens Health. 2019;28(1):46-52. doi:10.1089/jwh.2018.7122.

25. Wang ET, Calderon-Margalit R, Cedars MI, et al. Polycystic ovary syndrome and risk for long-term diabetes and dyslipidemia. Obstet Gynecol. 2011;117(1):6-13. doi:10.1097/AOG.0b013e31820209bb.

26. Aksoy Y, Sivri N, Karaoz B, et al. Carotid intima-media thickness: A new marker of patients with uterine leiomyoma. J Obstet Gynecol Reprod Biol (Europe). 2014;175:54-7.

27. He Y, Zeng Q, Li X, Liu B, Wang P. The association between subclinical atherosclerosis and uterine fibroids. PLoS One, 2013; 8(2). doi:10.1371/journal.pone.0057089.

28. Lee SS, Kim DH, Nam GE, et al. Association between metabolic syndrome and menstrual irregularity in middle-aged Korean women. J Fam Med (Korea). 2016;37(1):31-6. doi:10.4082/kjfm.2016.37.1.31.

29. Rostami DM, Ramezani TF, Djalalinia S, et al. Menstrual cycle irregularity and metabolic disorders: A population-based prospective study. PLoS One, 2016; 11(12). doi:10.1371/journal.pone.0168402. 
30. Solomon CG, Hu FB, Dunaif A, et al. Long or highly irregular menstrual cycles as a marker for risk of type 2 diabetes mellitus. JAMA. 2001;286(19):2421-6. doi:10.1001/jama.286.19.2421.

31. Solomon CG, Hu FB, Dunaif A, et al. Menstrual cycle irregularity and risk for future cardiovascular disease. J Clin Endocrinol Metab. 2002;87:2013-7. doi:10.1210/jcem.87.5.8471.

32. National Institute of Diabetes and Digestive and Kidney Diseases. Overweight \& Obesity Statistics. https://www.niddk.nih.gov/health-information/health-statistics/overweight-obesity. Accessed Dec 26, 2017.

33. World Health Organization. Waist circumference and waist-hip ratio: Report of a WHO expert consultation. Geneva; 2008.

34. Ghasemi A, Tohidi M, Derakhshan A, et al. Cut-off points of homeostasis model assessment of insulin resistance, beta-cell function, and fasting serum insulin to identify future type 2 diabetes: Tehran lipid and glucose study. Acta Diabetol. 2015;52:905-15. doi:10.1007/s00592-015-0730-3.

35. Lee S, Choi S, Kim HJ, et al. Cutoff values of surrogate measures of insulin resistance for metabolic syndrome in Korean non-diabetic adults. J Korean Med Sci. 2006;21(4):695-700. doi:10.3346/jkms.2006.21.4.695.

36. Grundy SM, Stone NJ, Bailey AL, et al. AHA/ACC/AACVPR/AAPA/ABC/ACPM/ADA/AGS/APhA/ASPC/NLA/PCNA guideline on the management of blood cholesterol: A report of the American College of Cardiology/American Heart Association Task Force on clinical practice guidelines. J Am Coll Cardiol, 2018; 139:e1082-143. doi:10.1161/CIR.0000000000000625.

37. R Core Team. R: A language and environment for statistical computing. Vienna: R Foundation for Statistical Computing; 2018. https://www.R-project.org.

38. Small CM, Manatunga AK, Marcus M. Validity of self-reported menstrual cycle length. Ann Epidemiol. 2007;17:163-70.

39. Meadows TA, Bhatt DL, Cannon CP, et al. Ethnic differences in cardiovascular risks and mortality in atherothrombotic disease: Insights from the Reduction of Atherothrombosis for Continued Health (REACH) registry. Mayo Clin Proc. 2011;86(10):960-7. doi:10.4065/mcp.2011.0010.

\section{Tables}

Due to technical limitations, table 1, 2, 3, 4 is only available as a download in the Supplemental Files section.

\section{Supplementary Files}

This is a list of supplementary files associated with this preprint. Click to download.

- Table1.png

- Table2.png 
- Table3.png

- Table4.png

Page $12 / 12$ 